\title{
RIEMANN SURFACES IN COMPLEX PROJECTIVE SPACES
}

\author{
BANG-YEN CHEN AND GERALD D. LUDDEN
}

\begin{abstract}
The complex projective line and the complex quadric are the only compact Riemann surfaces in the complex projective plane with constant scalar normal curvature.
\end{abstract}

1. Introduction. Let $M^{n}$ be an $n$-dimensional manifold immersed in an $(n+p)$-dimensional Riemannian manifold $R^{n+p}$. The scalar normal curvature $K_{N}$ of $M^{n}$ in $R^{n+p}$ is defined as the length of the curvature form of the connection in the normal bundle (for the details; see §2). It seems to the authors that the scalar normal curvature is important for the theory of submanifolds of higher codimensions. In [1], [2], [4], Itoh and the authors studied the scalar normal curvature for submanifolds of real space forms. In the present paper, we shall study the scalar normal curvature for submanifolds of a Kähler manifold. In particular, we have

MaIn TheOREM. The complex projective line $C P^{1}$ and the complex quadric $Q^{1}$ are the only compact Riemann surfaces in the complex projective plane with constant scalar normal curvature.

2. Local formulas. In this section, we shall compute some elementary formulas for later use and define the scalar normal curvature.

Let $M$ be an $n$-dimensional manifold immersed in an $(n+p)$-dimensional Riemannian manifold $N^{n+p}$. We choose a local field of orthonormal frames $\boldsymbol{e}_{1}, \cdots, \boldsymbol{e}_{n+p}$ in $N^{n+p}$ such that, restricted to $M$, the vectors $\boldsymbol{e}_{1}, \cdots, \boldsymbol{e}_{n}$ are tangent to $M$ (and, consequently, $e_{n+1}, \cdots, e_{n+p}$ are normal to $M$ ). We shall make use of the following convention on the ranges of indices:

$$
\begin{aligned}
1 & \leqq A, B, C, \cdots \leqq n+p ; \quad 1 \leqq i, j, k, \cdots \leqq n ; \\
n+1 & \leqq \alpha, \beta, \gamma, \cdots \leqq n+p ;
\end{aligned}
$$

and we shall agree that repeated indices are summed over the respective ranges. With respect to the frame field of $N^{n+p}$ chosen above, let $\omega^{1}, \cdots$, $\omega^{n+p}$ be the field of dual frames. Then the structure equations of $N^{n+p}$ are

Received by the editors July 23, 1971.

AMS 1970 subject classifications. Primary 53B35, 53C40, 53C55; Secondary 32J15.

Key words and phrases. Complex projective space, complex quadric, scalar normal curvature, Kähler manifold, second fundamental form.

(c) American Mathematical Society 1972 
given by

$$
\begin{gathered}
d \omega^{A}=-\sum \omega_{B}^{A} \wedge \omega^{B}, \quad \omega_{B}^{A}+\omega_{A}^{B}=0 \\
d \omega_{B}^{A}=-\sum \omega_{C}^{A} \wedge \omega_{B}^{C}+\Phi_{B}^{A}, \quad \Phi_{B}^{A}=\frac{1}{2} \sum K_{B C D}^{A} \omega^{C} \wedge \omega^{D}, \\
K_{B C D}^{A}+K_{B D C}^{A}=0 .
\end{gathered}
$$

We restrict these forms to $M$. Then $\omega^{\alpha}=0$. Since $0=d \omega^{\alpha}=-\sum \omega_{i}^{\alpha} \wedge \omega^{i}$, by Cartan's lemma we may write

$$
\omega_{i}^{\alpha}=\sum h_{i j}^{\alpha} \omega^{j}, \quad h_{i j}^{\alpha}=h_{j i}^{\alpha} .
$$

From these formulas, we obtain

$$
\begin{aligned}
d \omega^{i} & =-\sum \omega_{j}^{i} \wedge \omega^{j}, \quad \omega_{j}^{i}+\omega_{i}^{j}=0, \\
d \omega_{j}^{i} & =-\sum \omega_{k}^{i} \wedge \omega_{j}^{k}+\Omega_{j}^{2}, \quad \Omega_{j}^{i}=\frac{1}{2} \sum R_{j k l}^{i} \omega^{k} \wedge \omega^{l}, \\
R_{j k l}^{i} & =K_{j k l}^{i}+\sum_{\alpha}\left(h_{i k}^{\alpha} h_{j l}^{\alpha}-h_{i l}^{\alpha} h_{j k}^{\alpha}\right), \\
d \omega_{\beta}^{\alpha} & =-\sum \omega_{\gamma}^{\alpha} \wedge \omega_{\beta}^{\gamma}+\Omega_{\beta}^{\alpha}, \quad \Omega_{\beta}^{\alpha}=\frac{1}{2} \sum R_{\beta k l}^{\alpha} \omega^{k} \wedge \omega^{l}, \\
R_{\beta k l}^{\alpha} & =K_{\beta k l}^{\alpha}+\sum_{i}\left(h_{i k}^{\alpha} h_{i l}^{\beta}-h_{i l}^{\alpha} h_{i k}^{\beta}\right) .
\end{aligned}
$$

We call $\sum h_{i j}^{\alpha} \omega^{i} \omega^{j} \boldsymbol{e}_{\alpha}$ the second fundamental form of the immersed manifold $M$. The mean curvature vector is given by $(1 / n) \sum_{\alpha}\left(\sum_{i} h_{i i}^{\alpha}\right) \boldsymbol{e}_{\alpha}$.

We denote by $K_{N}$ the length of the curvature form of the normal connection, i.e.,

$$
K_{N}=\sum S_{\beta i j}^{\alpha} S_{\beta i j}^{\alpha}, \quad S_{\beta i j}^{\alpha}=\sum\left(h_{i k}^{\alpha} h_{j k}^{\beta}-h_{j k}^{\alpha} h_{i k}^{\beta}\right) .
$$

We call $K_{N}$ the scalar normal curvature of this immersion.

The forms $\left(\omega_{j}^{i}\right)$ define a connection in the tangent bundle $T=T(M)$ and the $\left(\omega_{\beta}^{\alpha}\right)$ define a connection in the normal bundle $T^{\perp}=T^{\perp}(M)$. Consequently, we have covariant differentiation which maps a section of $T^{\perp} \otimes T^{*} \otimes \cdots \otimes T^{*},\left(T^{*} ; k\right.$ times $)$, into a section of $T^{\perp} \otimes T^{*} \otimes \cdots \otimes T^{*} \otimes T^{*}$, $\left(T^{*} ; k+1\right.$ times). The second fundamental form $h_{i j}^{\alpha}$ is a section of the vector bundle $T^{\perp} \otimes T^{*} \otimes T^{*}$.

We take exterior differentiation of (2.3) and define $h_{i j k}^{\alpha}$ by

$$
\sum h_{i j k}^{\alpha} \omega^{k}=d h_{i j}^{\alpha}-\sum h_{i l}^{\alpha} \omega_{j}^{l}-\sum h_{l j}^{\alpha} \omega_{i}^{l}+\sum h_{i j}^{\beta} \omega_{\beta}^{\alpha} .
$$

Then

$$
\sum\left(h_{i j k}^{\alpha}+\frac{1}{2} K_{i j k}^{\alpha}\right) \omega^{j} \wedge \omega^{k}=0
$$


We take exterior differentiation of $(2.10)$ and define $h_{i j k l}^{\alpha}$ by

$$
\sum h_{i j k l}^{\alpha} \omega^{l}=d h_{i j k}^{\alpha}-\sum h_{l j k}^{\alpha} \omega_{i}^{l}-\sum h_{i l k}^{\alpha} \omega_{j}^{l}-\sum h_{i j l}^{\alpha} \omega_{k}^{l}+\sum h_{i j k}^{\beta} \omega_{\beta}^{\alpha} .
$$

The Laplacian $\Delta h_{i j}^{\alpha}$ of the second fundamental form $h_{i j}^{\alpha}$ is defined by

$$
\Delta h_{i j}^{\alpha}=\sum_{k} h_{i j k k}^{\alpha} \text {. }
$$

By a straightforward, simple calculation we have [3]

$$
\begin{aligned}
\Delta h_{i j}^{\alpha}= & \sum_{k} h_{k k i j}^{\alpha}+\sum_{\beta, k}\left(-K_{i j \beta}^{\alpha} h_{k k}^{\beta}+2 K_{\beta k i}^{\alpha} h_{j k}^{\beta}-K_{k \beta k}^{\alpha} h_{i j}^{\beta}+2 K_{\beta k j}^{\alpha} h_{k i}^{\beta}\right) \\
& +\sum_{m, k}\left(K_{k i k}^{m} h_{m j}^{\alpha}+K_{k j k}^{m} h_{m i}^{\alpha}+2 K_{i j k}^{m} h_{m k}^{\alpha}\right) \\
& +\sum_{\beta, m, k}\left(h_{m i}^{\alpha} h_{m j}^{\beta} h_{k k}^{\alpha}+2 h_{k m}^{\alpha} h_{k i}^{\beta} h_{m j}^{\beta}-h_{k m}^{\alpha} h_{k m}^{\beta} h_{i j}^{\beta}\right. \\
& \left.-h_{m i}^{\alpha} h_{m k}^{\beta} h_{k j}^{\beta}-h_{m j}^{\alpha} h_{k i}^{\beta} h_{m k}^{\beta}\right) .
\end{aligned}
$$

3. Proof of Main Theorem. Let $C P^{m}$ be the complex projective space of complex dimension $m=1+p / 2$ with the Fubini-Study metric. Let $M$ be a compact Riemann surface holomorphically immersed in $C P^{m}$, and $J$ be the complex structure of $C P^{m}$. Then $M$ is a minimal submanifold in $C P^{m}$ and the curvature tensor of $C P^{m}$ is given by

$$
K_{B C D}^{A}=\frac{1}{4}\left(\delta_{A C} \delta_{B D}-\delta_{A D} \delta_{B C}+J_{A C} J_{B D}-J_{A D} J_{B C}+2 J_{A B} J_{C D}\right) \text {. }
$$

By a straightforward, simple calculation, formula (2.23) of [3] reduces to

$$
\begin{aligned}
\sum h_{i j}^{\alpha} \Delta h_{i j}^{\alpha}= & -\sum\left(h_{i k}^{\alpha} h_{k j}^{\beta}-h_{i k}^{\beta} h_{k j}^{\alpha}\right)\left(h_{i l}^{\alpha} h_{l j}^{\beta}-h_{i l}^{\beta} h_{l j}^{\alpha}\right) \\
& -\sum h_{i j}^{\alpha} h_{k l}^{\alpha} h_{i j}^{\beta} h_{k l}^{\beta}+\frac{3}{2} \sum h_{i j}^{\alpha} h_{i j}^{\alpha} .
\end{aligned}
$$

Similarly, formula (2.22) of [3] gives

$$
\begin{aligned}
\frac{1}{2} \Delta K_{N}= & \sum\left(S_{\beta i j k}^{\alpha}\right)^{2}+2 \sum S_{\beta i j}^{\alpha}\left(h_{i k l}^{\alpha} h_{j k l}^{\beta}-h_{i k l}^{\beta} h_{j k l}^{\alpha}\right)+4 \sum S_{\beta i j}^{\alpha}\left(\Delta h_{i k}^{\alpha}\right) h_{j k}^{\beta} \\
= & \sum\left(S_{\beta i j k}^{\alpha}\right)^{2}+2 \sum S_{\beta i j}^{\alpha}\left(h_{i k l}^{\alpha} h_{j k l}^{\beta}-h_{i k l}^{\beta} h_{j k l}^{\alpha}\right) \\
& +\left(3-\sum h_{i j}^{\alpha} h_{i j}^{\alpha}\right) K_{N}+8 \sum S_{\beta i j}^{\alpha} h_{l m}^{\alpha} h_{m k}^{\gamma} h_{i l}^{\gamma} h_{j k}^{\beta} \\
& -4 \sum S_{\beta i j}^{\alpha}\left[\sum h_{l m}^{\alpha} h_{l m}^{\gamma} h_{i k}^{\gamma} h_{j k}^{\beta}+\sum h_{m k}^{\alpha} h_{l i}^{\gamma} h_{m l}^{\gamma} h_{j k}^{\beta}\right] .
\end{aligned}
$$

For a matrix $A=\left(a_{i j}\right)$ we denote by $N(A)$ the square of the norm of $A$, i.e.,

$$
N(A)=\operatorname{trace} A \cdot{ }^{t} A=\sum\left(a_{i j}\right)^{2} .
$$

For each $\alpha$, let $H_{\alpha}$ denote the symmetric matrix $\left(h_{i j}^{\alpha}\right)$ and set

$$
S_{\alpha}=N\left(H_{\alpha}\right), \quad S=\sum S_{\alpha} .
$$


Then (3.1) reduces to

$$
\sum h_{i j}^{\alpha} \Delta h_{i j}^{\alpha}=-\sum N\left(H_{\alpha} H_{\beta}-H_{\beta} H_{\alpha}\right)-\sum S_{\alpha}^{2}+\frac{3}{2} S .
$$

From now on, we assume that the scalar normal curvature $K_{N}$ of $M$ in $C P^{m}$ is constant. We now consider the cases $K_{N}=0$ and $K_{N} \neq 0$ separately.

Case $K_{N} \neq 0$. We define a linear mapping $\varphi$ from the normal space $T_{p}^{\perp}$ at $p$ into the space of all symmetric matrices of order 2 by

$$
\varphi\left(\sum \nu_{\alpha} \boldsymbol{e}_{\alpha}\right)=\sum \nu_{\alpha} H_{\alpha}
$$

Set $O_{p}=\varphi^{-1}(0)$. Then by the assumption $K_{N} \neq 0$ and $\sum h_{i i}^{\alpha}=0$, we see that $\operatorname{dim} O_{p}=2 m-4$. Let $N_{p}^{\prime}$ be the subspace of $T_{p}^{\perp}$ given by

$$
T_{p}^{\llcorner}=N_{p}^{\prime} \oplus O_{p}, \quad N_{p}^{\prime} \perp O_{p} .
$$

Then $\operatorname{dim} N_{p}^{\prime}=2$ and this decomposition is well defined and smooth. In the following, we always choose $e_{3}, \boldsymbol{e}_{4} \in N_{p}^{\prime}$. Then we have

$$
h_{i j}^{\gamma}=0 \text { for } \gamma>4
$$

Hence, (3.3) reduces to

$$
\begin{aligned}
\frac{1}{2} \Delta K_{N}= & 2 \sum\left(S_{\beta i j k}^{\alpha}\right)^{2}+4 \sum S_{4 i j}^{3}\left(h_{i k l}^{3} h_{j k l}^{4}-h_{i k l}^{4} h_{j k l}^{3}\right) \\
& +(3-S) K_{N}-2 \operatorname{trace}\left(R H_{3}-H_{3} R\right)^{2} \\
& -2 \operatorname{trace}\left(R H_{4}-H_{4} R\right)^{2},
\end{aligned}
$$

where $R=\left(S_{4 i j}^{3}\right)$. We choose $e_{1}, e_{2}$ in the principal directions of $e_{3}$ and $e_{2}=J e_{1}$. Then we have

$$
\begin{gathered}
H_{3}=\left[\begin{array}{cc}
a & 0 \\
0 & -a
\end{array}\right], \quad H_{4}=\left[\begin{array}{cc}
d & e \\
e & -d
\end{array}\right], \quad a \neq 0, d^{2}+e^{2} \neq 0 \\
R=\left(S_{4 i j}^{3}\right)=\left[\begin{array}{cc}
0 & 2 a e \\
-2 a e & 0
\end{array}\right] \\
S=2 a^{2}+2 d^{2}+2 e^{2} \\
K_{N}=16 a^{2} e^{2}=\text { constant }>0 \\
\operatorname{trace}\left(R H_{3}-H_{3} R\right)^{2}=32 a^{4} e^{2}=2 a^{2} K_{N} \\
\operatorname{trace}\left(R H_{4}-H_{4} R\right)^{2}=2\left(d^{2}+e^{2}\right) K_{N}
\end{gathered}
$$

Hence, (3.8) reduces to

(3.15) $\frac{1}{2} \Delta K_{N}=4 \sum S_{4 i j}^{3}\left(h_{i k l}^{3} h_{j k l}^{4}-h_{i k l}^{4} h_{j k l}^{3}\right)+3(1-S) K_{N}+\sum\left(S_{\beta i j k}^{\alpha}\right)^{2}$. 
If $m=2$, then from (3.12) and (3.15) we obtain

$$
K_{N}(1-S)=\frac{32}{3} \sum_{l}\left(a_{, l} e\right)^{2} \geqq 0,
$$

where $a_{, l}$ denotes the covariant derivative of $a$. By Lemma 1 of [3], (3.5) and (3.16) we obtain

$$
\begin{aligned}
\sum h_{i j}^{\alpha} \Delta h_{i j}^{\alpha} & \geqq-S_{3}^{2}-S_{4}^{2}+\frac{3}{2} S-4 S_{3} S_{4} \\
& =\frac{3}{2}(1-S) S+\frac{1}{2}\left(S_{3}-S_{4}\right)^{2} \\
& \geqq \frac{1}{2}\left(S_{3}-S_{4}\right)^{2} \geqq 0 .
\end{aligned}
$$

Hence we obtain

$$
\frac{1}{2} \Delta S=\sum\left(h_{i j k}^{\alpha}\right)^{2}+\sum h_{i j}^{\alpha} \Delta h_{i j}^{\alpha} \geqq 0 .
$$

By Hopf's lemma, we see that $S$ is constant and

$$
\begin{gathered}
h_{i j k}^{\alpha}=0, \\
\sum h_{i j}^{\alpha} \Delta h_{i j}^{\alpha}=0 .
\end{gathered}
$$

Formula (3.20) implies

$$
\begin{gathered}
N\left(H_{3} H_{4}-H_{4} H_{3}\right)^{2}=2 N\left(H_{3}\right) N\left(H_{4}\right), \\
S_{3}=S_{4}=\frac{1}{2} .
\end{gathered}
$$

Therefore, by Lemma 1 of [3] we see that $H_{3}$ and $H_{4}$ are scalar multiples of

$$
\tilde{A}=\left[\begin{array}{rr}
1 & 0 \\
0 & -1
\end{array}\right], \quad \tilde{B}=\left[\begin{array}{ll}
0 & 1 \\
1 & 0
\end{array}\right]
$$

respectively. Hence, we may conclude that $d=0$ and

$$
H_{3}=\left[\begin{array}{rr}
\frac{1}{2} & 0 \\
0 & -\frac{1}{2}
\end{array}\right], \quad H_{4}=\left[\begin{array}{ll}
0 & \frac{1}{2} \\
\frac{1}{2} & 0
\end{array}\right] \text {. }
$$

On the other hand, since $e_{2}=J e_{1}$, we can easily show that the second fundamental form in the direction of $J e_{3}$ is given by

Since $h_{i j k}^{\alpha}=0$, we have

$$
\left[\begin{array}{rr}
0 & -\frac{1}{2} \\
-\frac{1}{2} & 0
\end{array}\right]
$$

$$
d h_{i j}^{\alpha}=\sum h_{i k}^{\alpha} \omega_{j}^{k}+\sum h_{k j}^{\alpha} \omega_{i}^{k}-\sum h_{i j}^{\beta} \omega_{\beta}^{\alpha} .
$$

Using (3.23) and (3.24) we can prove that

$$
\omega_{3}^{4}=2 \omega_{1}^{2} .
$$

Therefore, we may conclude that $M$ is a complex quadric in $C P^{2}$. 
Case $K_{N}=0$. This case is contained in the following:

Proposition. Let $M$ be a complex submanifold of a Kähler manifold $\tilde{M}$ with complex structure $J$. Then the scalar normal curvature $K_{N}=0$ at $p \in M$ if and only if the second fundamental form $h=0$ at $p$.

Proof. Let $A$ be the second fundamental form in the direction $e$. Then if $K_{N}=0$ at $p$, we have

$$
N(A J A-J A A)=0
$$

This implies that

$$
\text { trace }\left(A J A^{t}(A J A)\right)=\operatorname{trace} A^{4}=0 .
$$

By (3.26) and $A={ }^{t} A$, we obtain $A=0$ at $p$. Consequently, $h=0$ at $p$. The converse of this is trivial. This proves the proposition. Hence the Main Theorem is proved completely.

\section{REFERENCES}

1. B.-Y. Chen, Pseudo-umbilical submanifolds of a Riemannian manifold of constant curvature. III, J. Differential Geometry (to appear).

2. B.-Y. Chen and G. D. Ludden, Surfaces with mean curvature vector parallel in the normal bundle (to appear).

3. S. S. Chern, M. do Carmo and S. Kobayashi, Minimal submanifolds of a sphere with second fundamental form of constant length, Functional Analysis and Related Fields, 1970, pp. 57-75.

4. T. Itoh, Minimal surfaces in 4-dimensional Riemannian manifold of constant curvature, Kōdai Math. Sem. Rep. (to appear).

Department of Mathematics, Michigan State University, East Lansing, MichiGAN 48823 